\title{
CUORE-0 and CUORE sensitivity to WIMPs and Supernova neutrinos
}

\author{
Elena Ferri on behalf of the CUORE collaboration* \\ Università di Milano-Bicocca and INFN Milano-Bicocca \\ E-mail: elena.ferri@mib.infn.it
}

The Cryogenic Underground Observatory for Rare Events (CUORE) is a bolometric experiment. CUORE is an array of $988 \mathrm{TeO}_{2}$ crystals of $750 \mathrm{~g}$ each arranged in a cylindrical compact and granular structure of 19 towers. The primary target of CUORE is the observation of Neutrinoless Double Beta Decay of ${ }^{130} \mathrm{Te}$. However, thanks to its high mass, the good energy resolution, and the low background the experiment can also be sensitive to other rare processes, such as Dark Matter and neutrinos emitted by core collapse Supernovae, that can be detected via the observation of the target nuclei recoil energy. In this contribution, the sensitivity of CUORE-0 -a prototype tower- and the sensitivity of CUORE to an annual signal modulation induced by WIMP Dark Matter candidates and to Supernova neutrinos are presented.

XV Workshop on Neutrino Telescopes,

11-15 March 2013

Venice, Italy

\footnotetext{
*Speaker.
} 


\section{Introduction}

CUORE (Cryogenic Underground Observatory for Rare Events) is a bolometric experiment searching for the Neutrinoless Double Beta Decay $(0 v \beta \beta)$ of ${ }^{130} \mathrm{Te}$ with cryogenic detectors. It will consist of $988 \mathrm{TeO}_{2}$ crystals, $750 \mathrm{~g}$ each, arranged in a cylindrical and granular structure of 19 towers. It is expected to reach a background level of the order of 0.01 counts $/ \mathrm{keV} / \mathrm{kg} / \mathrm{y}$ at the ${ }^{130} \mathrm{Te}$ Q-value (around $2528 \mathrm{keV}[3,4,5]$ ), allowing a sensitivity to $0 v \beta \beta$ down to the inverted hierarchy region of neutrino masses. CUORE is currently being assembled at the Laboratori Nazionali del Gran Sasso (LNGS) in Italy and it is expected to go into operation at the end of 2014. Its feasibility was demonstrated by Cuoricino, an array of 62 crystals of $\mathrm{TeO}_{2}$ with a total mass of $40.7 \mathrm{~kg}$, which was running from February 2003 until June 2008 in Hall A of LNGS. Cuoricino put stringent limits on the $0 v \beta \beta$ half-life of ${ }^{130} \mathrm{Te}[6,7]$. To test the CUORE mechanical procedures such as material selection, cleaning and gluing, an array of $52 \mathrm{TeO}_{2}$ bolometers, CUORE- 0 , has been built and it is now in data taking.

Thanks to their high mass, the good energy resolution (few keV over a wide energy range extending from a few $\mathrm{keV}$ up to several $\mathrm{MeV}$ ) and the low background, CUORE and CUORE-0 can also be sensitive to other rare processes. Indeed Dark Matter and neutrinos emitted by core collapse Supernovae can be detected via the observation of the target nuclei recoil energy. In this contribution, the sensitivity of CUORE-0 and the sensitivity of CUORE to an annual modulation signal induced by WIMP Dark Matter candidates and to Supernova neutrinos are presented.

\section{Sensitivity to Dark Matter}

One of the most exciting problems in astrophysics and cosmology, but also one of the most attractive connection points of these fields to elementary particle physics is the explanation of the Dark Matter (DM). In fact, although there is significant evidence that Dark Matter (DM) exists [8], its nature remains still elusive. Weakly Interacting Massive Particles (WIMPs) are a possible DM candidate and several experiments have been designed to detect their scattering off nuclei, which should produce energy releases of a few $\mathrm{keV}$ [9]. WIMPs can couple to nucleons via both spinindependent and spin-dependent (axial vector) interactions. Spin-independent scattering dominates when the mass number $\mathrm{A}>30$, so that $\mathrm{TeO}_{2}$ bolometers will be most sensitive for spin-independent interacting WIMPs. DM searches were not performed in Cuoricino because the energy threshold was too high (order of tens of keV). To lower the energy threshold down to the energy where the modulation signal is expected a new software trigger was developed [10]. The trigger is based on the Optimum Trigger [11, 12], that is applied to the continuous data flow. The trigger also provides a pulse shape parameter to suppress events generated by electronic noise and detector vibrations. To estimate the sensitivity to WIMPs for CUORE-0 and CUORE the background rate of these experiments at low energy $(3-25 \mathrm{keV})$ was assumed to be equal to the one measured in the CUORE Crystal Validation Run 2 (CCVR2) [13], in which the dedicated trigger was applied to lower the threshold at $3 \mathrm{keV}$. Then to evaluate the $90 \% \mathrm{CL}$ upper limit sensitivity to the crosssection as a function of the WIMP mass, toy Monte Carlo simulations are performed generating background events according to the CCVR2 fit functions, and WIMP events according to the theoretical distribution described in [14], using the following WIMP parameters: density $\rho_{W}=0.3$ 


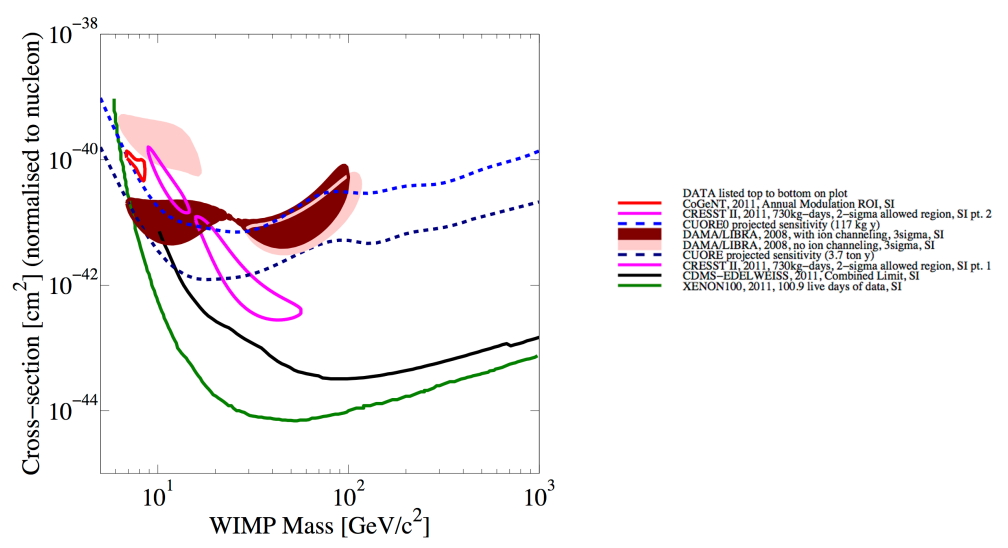

Figure 1: $90 \%$ sensitivity to WIMP spin-independent scattering of CUORE-0 and CUORE experiments assuming a $3 \mathrm{keV}$ threshold for all detectors and the same background level of the CCVR2 detectors. The evidences of DAMA-3 $\sigma$ [17], CoGeNT-90\% [18] and CRESST-2 $\sigma$ [19] are also reported for comparison.

$\mathrm{GeV} / \mathrm{cm}^{3}$, average velocity $\mathrm{v}_{0}=220 \mathrm{~km} / \mathrm{s}$ and escape velocity from the Galaxy $\mathrm{v}_{\text {esc }}=600 \mathrm{~km} / \mathrm{s}$ [15]. A quenching factor for nuclear recoils in $\mathrm{TeO}_{2}$ equal to 1 was used [16]. The results are reported in figure 1 for 3-years of CUORE-0 data taking and 5-years of CUORE. The comparison with other experiments shows that CUORE could test the DAMA results.

\section{Sensitivity to Supernova Neutrino}

Core-collapse Supernovae emit a large quantity of neutrinos. These neutrino fluxes carry information not only on the neutrino nature but also on the Supernova mechanism. Furthermore, their detection constitutes the only available prompt detectable signal of the Supernova explosion itself. CUORE could detect Supernova neutrinos via coherent scattering on Te and very marginally $\mathrm{O}$ nuclei, through the measure of their recoil energy from few to tens of keV. Also in this case a very low threshold of few $\mathrm{keV}$ is mandatory to detect this kind of interactions.

A Supernova trigger algorithm is implemented in the official CUORE DAQ system. Since the Supernova occurrence probability is extremely small compared with the rate of events occurring in the energy region in which its presence is searched for (the rate of low energy interactions in the detector is expected to be of $1 \mathrm{~Hz}$ on the basis of [13]), a threshold on the signal magnitude must be set on the basis of a false-positive rate criterion. A toy Monte Carlo simulation of the fit-derived signal is needed to select the threshold that corresponds to the desired false-positive probability [20]. The discovery power corresponding to a rate of false positive of $1 /$ week is calculated by simulating the signal at different distances as shown in figure 2. For example, a Supernova at $6 \mathrm{kPc}$ is triggered with $95 \%$ efficiency, while a Supernova at $7.2 \mathrm{kPc}$ with $50 \%$ efficiency.

\section{Conclusions}

Regards the detection of WIMPs CUORE could investigate the same parameter space of the DAMA/LIBRA experiment, and could look for an annual modulation of dark matter interactions. CUORE could also be sensitive to Supernova neutrinos and for this reason a Supernova trigger 


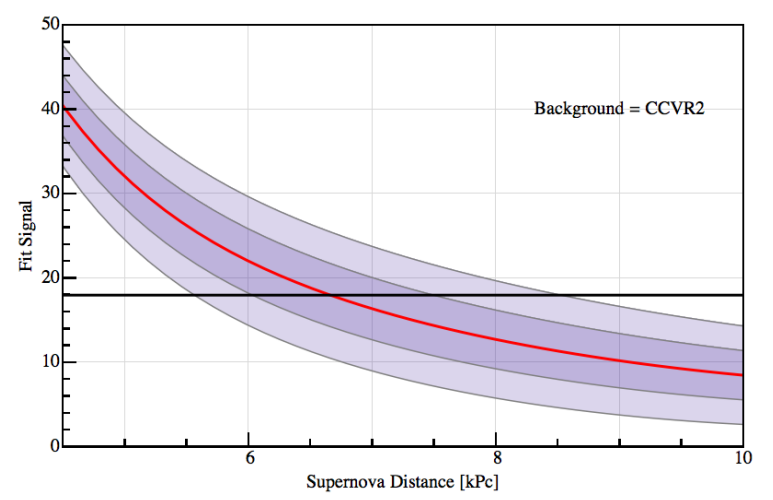

Figure 2: The expected signal as a function of the Supernova distance for the CUORE experiment. Shadowed bands are $1 \sigma(2 \sigma)$ signal fluctuations. The black line is the threshold that corresponds to a false positive rate of one per week.

algorithm is implemented in the official CUORE DAQ system with this method. A Supernova at 6 $\mathrm{kPc}$ is triggered with $95 \%$ efficiency.

\section{References}

[1] C. Arnaboldi et al., Astropart. Phys. 2091 (2003).

[2] C. Arnaboldi et al., Nucl. Instrum. Meth. A 518775 (2004).

[3] M. Redshaw, B.J. Mount, E.G. Myers and F.T. Avignone, Phys. Rev. Lett. 102212502 (2009).

[4] N. Scielzo et al., Phys. Rev. C 80025501 (2009).

[5] S. Rahaman et al., Phys. Lett. B 703412 (2011).

[6] E. Andreotti et al., Astropart. Phys. 34822 (2011).

[7] E. Andreotti et al., it Phys. Rev. C 85045503 (2012).

[8] G. Bertone, D. Hooper and J. Silk, Phys. Rept. 405279 (2005).

[9] M.W. Goodman and E. Witten, Phys. Rev. D 31 (1985) 3059 [INSPIRE].

[10] S. di Domizio et al, JINST 6 P02007 (2011).

[11] E. Gatti and P.F. Manfredi, Riv. Nuovo Cimento 91 (1986).

[12] V. Radeka and N. Karlovac, Nucl. Instrum. Meth. 5286 (1967).

[13] F. Alessandria et al, Astro. Phys. 12839 (2012).

[14] F. Alessandria et al., JCAP01 038 (2013).

[15] J. D. Lewin and P. F. Smithl, Astrop. Phys. 687 (1996).

[16] A. Alessandrello et. al., NIMA 409451 (1998).

[17] R. Bernabei et al., Eur. Phys. J. C 56333 (2008).

[18] C. Aalseth et al., Phys. Rev. Lett. 107141301 (2011).

[19] G. Angloher et al., Eur. Phys. J. C 721971 (2012).

[20] M. Biassoni, il NUOVO CIMENTO 36 C n. 1 (2013). 